\title{
A comparison of follow-up regimes in rheumatoid arthritis
}

\author{
A. G. MOWAT, ${ }^{1}$ P. J. R. NICHOLS, ${ }^{2}$ E. M. HOLLINGS, ${ }^{1}$ \\ R. J. HAWORTH, ${ }^{2}$ AND L. C. AITKEN ${ }^{2}$
}

From the ${ }^{1}$ Rheumatology Unit, Nuffield Orthopaedic Centre, and ${ }^{2}$ Oxford Rehabilitation Research Unit, Nuffield Orthopaedic Centre, Oxford

SUMMARY One hundred and thirty-two patients discharged from a rheumatology unit were randomly allocated to general practitioner care, attendance at hospital outpatient clinics, or follow-up by a senior occupational therapist attached to the hospital treatment team. At the end of 1 and 2 years a number of clinical and functional tests were applied, and information was gathered about the provision and use of aids and the provision of domestic support. In addition the standard of overall care was judged by an independent assessor. Although no significant intergroup differences in disease activity or function emerged, it is clear that patients prefer continuing contact with the hospital team, and this may lead to differences appearing in the future. The financial advantages of therapist follow-up are discussed.

The wide spectrum of clinical expression of rheumatoid arthritis and the variable progress of the disease in an individual patient mean that therapeutic efforts and advice to the patient based on a single examination and an assessment of the level of pain and disability are an unacceptable form of management. Many patients need frequent reassessments of their disease and disability and hence changes in their management. Consequently, in a number of centres the availability of a steadily increasing range of drugs, some of which can alter the natural history of the disease, the important contribution of orthopaedic surgery, the wide range of aids and appliances available, and the increasing social and economic support extended to the disabled have led to the concept of a team approach to the management of patients with rheumatoid arthritis. Further, increased public and medical awareness that something can be done for most patients has led to more referrals to hospital outpatient clinics, while many general practitioners request that the patients should continue to attend hospital departments so that they can be reassessed and decisions be made about the often complex therapeutic alternatives. Such trends have inevitably increased the strain

Accepted for publication 11 January 1979

Correspondence to Dr A. G. Mowat, Department of Rheumatology, Nuffield Orthopaedic Centre, Headington, Oxford OX3 7LD. on outpatient departments and reduced the time available for assessment of and advice to individual patients.

Although the emergence of better prognostic indicators of disease progress (Feigenbaum et al., 1977) and the increase in postgraduate education will allow those with milder disease to be identified and adequately treated in the community, the need remains for better utilisation of the limited hospital team expertise. Further, there are cogent arguments for reducing the expense of hospital attendance and for increasing the value of assessment methods by seeing the patients, most of whom are housewives, in their own homes.

Home visiting by one or more paramedical workers attached to the hospital team has been advocated by several groups (Vignos et al., 1972; Firth et al., 1973; Brattstrom, 1975). This paper compares the results of a 2-year follow-up by the general practitioner, home visiting by a senior occupational therapist attached to the hospital team, and attendance at hospital outpatient department of patients with rheumatoid arthritis after their discharge from a rheumatology unit.

\section{Materials}

One hundred and thirty-two patients (25 men, 107 women) with definite rheumatoid arthritis who had 
been treated in a rheumatology unit for a minimum of 14 days were entered into the study. Patients were excluded if their treatment with special drugs or new operative procedures required close supervision by the unit and if they lived outside a 50 -mile $(80-\mathrm{km})$ radius of the hospital. Patients were randomly allocated to 1 of 3 groups just before discharge from the ward.

Group 1: general practitioner follow-up. The general practitioner was warned before discharge of our intention; the patient returned for further assessment and advice only on request.

Group 2: routine hospital outpatient follow-up. This was undertaken during the usual clinic hours and the patient was not especially identified or necessarily seen by the same member of staff. The patient attended as often as considered necessary, usually at 3-monthly intervals.

Group 3: therapist follow-up. A senior occupational therapist attached to the unit visited the patient at home 10 days after discharge and then at 3-monthly intervals, further visits being allowed if it seemed necessary. The therapist undertook, so far as possible, the total follow-up of the patient. She was able to check with hospital staff and general practitioners on various aspects of management, but the patient returned to hospital only when the therapist or practitioner requested.

\section{Methods}

The patients were assessed on entering the trial and again at 1 and 2 years. The assessment covered the following: (a) a clinical evaluation, including articular index (Camp, 1971), by the consultant rheumatologist; (b) haemoglobin value, erythrocyte sedimentation rate, and rheumatoid factor test; (c) a standardised 37-item evaluation of the patient's functional level in activities of daily living covering 4 main categories - mobility, personal care, domestic activities, and social activities; $(d)$ a description of the patient's home environment; $(e)$ a questionnaire to assess patient's knowledge of his/her disease (entry and 1 year only); $(f)$ information on aids and appliances supplied and whether or not they were in use (entry and 1 year only); ( $g$ ) a subjective evaluation of the patient's management over the last year, her attitude to the disease, and the effect it had on her family life; $(h)$ a psychological assessment. This is the subject of a separate report.

The initial assessment was made by the rheumatologist and therapist on the ward before discharge. At the end of 1 or 2 years the patient was evaluated at home by an independent assessor using measurements $c-g$ who had no knowledge of the patient's group or social background. In addition the patient attended the ward for clinical assessment and blood tests $(a$ and $b)$.

\section{Results}

\section{PATIENTS}

Entry. On entry to the trial the 3 patient groups showed no significant difference in age, sex, disease duration, the number of previous admissions to the unit, or in any of the factors being measured in the study. As expected, the men had significantly higher haemoglobin values $(<0.001)$ and titres of rheumatoid factor $(\mathrm{P}<0.05)$. The men also had significantly less knowledge of their disease $(P<0.05)$. In all groups there was a decline in disease knowledge $(P<0.05)$ and an increase in erythrocyte sedimentation rate (ESR) and articular index (AI) with advancing age $(P<0.05)$. The ESR and $A I$ were higher in seropositive patients, and the values showed an inverse correlation with haemoglobin values $(\mathrm{P}<0 \cdot 01)$.

One year. One hundred and nineteen patients were available for review at 1 year. Thirteen patients had withdrawn (Table 1), the withdrawals being equally distributed between the groups.

Two years. Seventy-two patients were available for review at 2 years. For various reasons the study ended early, but there is no reason to believe from analysis of the data that these patients were unrepresentative of the whole group. Again there were 13 withdrawals (Table 1 ).

Articular index. At 1 year there were no overall or intergroup changes. In the second year, although none of the groups showed a significant change from its baseline value, group 3 patients showed a significant reduction $(P<0.05)$ compared with the other 2 groups.

Haemoglobin value. During the first year there was an overall increase in haemoglobin value, with the value being sustained during the second year of the study. There were no intergroup differences.

$E S R$. There were no overall or intergroup differences during either first or second year.

Rheumatoid factor tests. There were no overall or intergroup changes during the first year, but men with persistently positive tests showed a significant

Table 1 Trial withdrawals

\begin{tabular}{lll}
\hline During year 1 & Died & 2 \\
& Moved away & 2 \\
& Refused assessment & 5 \\
& Special rheumatoid surgery & 2 \\
& Other major surgery & 1 \\
During year 2 & Allocated wrong group & 1 \\
& Died & 4 \\
& Special rheumatoid drug therapy & 5 \\
& Special rheumatoid surgery & 1 \\
& Change to outpatient group & 3 \\
\hline
\end{tabular}


decrease in titre $(P<0.001)$. There were no changes during the second year.

Functional capacity (Table 2). Functional capacity was evaluated initially by the ward therapist and subsequently by the independent assessor using a 4-category proforma. The information was incorporated into scales of disability in which a value of 0 represented no function and 1 full function. The scales, which satisfied the Guttmann criteria of unidimensionality and accumulativeness (Williams et al., 1976), were designed so that a given disability was consistently associated with other related disabilities, so that, for example, the inability to perform function 5 in a scale meant that it could be predicted that functions 1-4 could also not be performed. The results were examined by an analysis of covariance, the regression of each group's scores being compared with baseline scores.

Observer error tests carried out during the development of the functional tests established the comparability and reproducibility of the various assessors' results.

At 1 year all 3 groups showed improvement in the mean value of the 4 scales, but significant improvement was found only in the scale for social activities for the general practitioner and therapists groups $(P<0.05)$. There were no intergroup differences. During the second year there were a number of minor changes, both of improvement and deterioration, with an overall trend for improvement, but there were no significant intergroup differences.

As reported elsewhere (Haworth and Hollings, in press), these functional gains are likely to be an underestimate. The patients in group 3 were additionally subjected to the $\mathbf{3 7}$ functional tests in their own homes by the visiting therapist 10 days after discharge from the unit and the scores compared with their predischarge scores. Significant decreases $(P<0.05)$ in the scores for 3 outdoor and 3 indoor activities were found, and smaller decreases were found in many others. No significant functional increase was found. Since the 3 patient groups were comparable, similar deterioration in function would be expected in each group. In consequence, the comparison of baseline function measured in hospital with function at 1 and 2 years measured at home fails to show the true extent of the improvement in each of the 3 patient groups.

\section{HOME ENVIRONMENT}

Isolation. On entry to the study 37 patients had problems of isolation, being alone for 7 or more hours each day, of whom 13 were living alone. These numbers did not change during the 2 years of the study, and there were no significant intergroup differences. On entry 53 patients $(40 \%)$ were registered as disabled with the social services department, and this rose to $61 \%$ at the end of the first year and $66 \%$ at the end of 2 years. Again there were no significant intergroup differences.

Work record. At the start of the study 19 men and 63 women were below retirement age, of whom 14 men and 19 women had full or part-time employment. During the 2 years there were no changes in the numbers in employment, but numbers of days off work in the first year decreased for all groups except for 4 men in group 2. During the first year there was a considerable increase in the numbers of patients with whom the disablement resettlement office was involved, and although this was not followed by an increase in the numbers employed the numbers of days off work decreased particularly in the second year, perhaps reflecting more suitable employment.

Disease knowledge. Use of the same questionnaire at entry and at 1 year showed there was a significant increase $(P<0.01)$ in disease knowledge. The greatest improvement occurred in those patients with lower initial scores, and this effect was increased in the younger patients. There were no sex or intergroup differences.

Visits. The number of patients in contact with a wide range of local authority and other visitors including health visitors, district nurses, domiciliary occupational therapists, social workers, chiropodists, home helps, and meals-on-wheels, and the frequency of the contacts, was established for the start of the trial and at 1 or 2 years. In addition attendances at day hospitals and a variety of clubs was also recorded. Significant increases in both the number of patients and the frequency of these contacts was found during the first year for all types

Table 2 Mean functional scale scores at entry, 1 and 2 years

\begin{tabular}{|c|c|c|c|c|c|c|c|c|c|}
\hline \multirow[t]{2}{*}{ Function } & \multicolumn{3}{|c|}{ GP group } & \multicolumn{3}{|c|}{ Outpatient group } & \multicolumn{3}{|c|}{ Therapist group } \\
\hline & Entry & 1 year & 2 years & Entry & 1 year & 2 years & Entry & 1 year & 2 years \\
\hline $\begin{array}{l}\text { Mobility } \\
\text { Personal care } \\
\text { Housekeeping } \\
\text { Social }\end{array}$ & $\begin{array}{l}0.59 \\
0 \cdot 71 \\
0 \cdot 54 \\
0 \cdot 39\end{array}$ & $\begin{array}{l}0.67 \\
0.76 \\
0.49 \\
0.58\end{array}$ & $\begin{array}{l}0.59 \\
0.68 \\
0.55 \\
0.60\end{array}$ & $\begin{array}{l}0.65 \\
0.70 \\
0.65 \\
0.53\end{array}$ & $\begin{array}{l}0.66 \\
0 \cdot 74 \\
0 \cdot 70 \\
0 \cdot 57\end{array}$ & $\begin{array}{l}0.72 \\
0 \cdot 74 \\
0.64 \\
0.60\end{array}$ & $\begin{array}{l}0.56 \\
0.73 \\
0.55 \\
0.45\end{array}$ & $\begin{array}{l}0.65 \\
0.75 \\
0.67 \\
0.59\end{array}$ & $\begin{array}{l}0.69 \\
0.83 \\
0.65 \\
0.68\end{array}$ \\
\hline
\end{tabular}


of contact. However, without exception the second year saw a return to pretrial values.

Exercises. At the end of 1 year it was found that 51 of the 94 patients were still performing the exercise programmes they had been given by the physiotherapist before discharge. At 2 years this had declined to 22 of 63 patients. However, many patients had been given very specific exercises, often to improve function at a single joint after surgery, and considered that they had achieved maximum improvement. There were no intergroup differences.

Aids and appliances. The numbers of aids and appliances in 15 different categories issued to the patients and actually in use at one year are shown in Table 3. This is the subject of a more detailed report (Hollings and Haworth, 1978). There were no significant differences in the usage rate by any group. However, while the numbers issued to group 1 and group 2 patients were similar at 284 and 260 respectively, group 3 patients had received 350 . Equal numbers of aids and applicances had been issued, many before the start of the trial, by the hospital (366) or domiciliary occupational therapy service (362), while the patients themselves had bought or devised 125. The remaining 41 aids had been provided by a vareity of agencies. There were no significant intergroup differences in the source of supply.

The greatest reduction in the use of aids and appliances was found in splints and related appliances such as cervical collars. This was largely expected, since many were intended only for shortterm support or as a functional aid. The change in usage of vehicles and adapted cars was accounted for by the non-use of 2 tricycles due to further deterioration in function, while the change in usage of stair and house access adaptations was

Table 3 Numbers of aids and appliances issued to 119 patients with rheumatoid arthritis and in use 1 year later

\begin{tabular}{lrrl}
\hline Aid or appliance & $\begin{array}{c}\text { Number } \\
\text { issued }\end{array}$ & $\begin{array}{c}\text { Number in } \\
\text { use }\end{array}$ & Percentage \\
\hline Splints and appliances & 170 & 101 & 59 \\
Walking aids & 77 & 56 & 73 \\
Wheelchairs & 22 & 20 & 91 \\
Vehicles or car & 7 & & \\
$\quad$ adaptations & 130 & 113 & 71 \\
Kitchen equipment & 37 & 35 & 87 \\
Stairs or access changes & 23 & 21 & 95 \\
House adaptations & 44 & 41 & 91 \\
Chairs and tables & 35 & 34 & 93 \\
Beds & 181 & 142 & 97 \\
Bath or shower changes & 81 & 68 & 78 \\
Toilet changes & 63 & 41 & 65 \\
ltems for personal care & 14 & 11 & 79 \\
Communication items & 10 & 8 & 80 \\
Others & 884 & 696 & \\
Total & &
\end{tabular}

simply explained by the discontinued use of doorkeys aids with improved hand function. The change in communication aids was the discontinued use of adapted pens, while most of the reduction in toilet aids was due to improved function reflected in non-use of raised toilet seats. Lastly, under house adaptations two outside toilets were not in use.

INDEPENDENT ASSESSMENT OF MANAGEMENT

Patients were asked to make an assessment of their care on a 5 point scale at the end of the first year. $59 \%$ of patients in the general practitioner group rated their care as above average compared with $87 \%$ of those in the outpatient group and $88 \%$ of those in the therapist group $(P<0.001)$. Similarly the independent assessor's evaluation of the patient's current rheumatological management on a 7 point scale showed that the percentages receiving above average care were $48.5 \%, 67 \%$, and $76 \%$ respectively. However, these findings were not reflected in the patients' rating of their rheumatoid arthritis or general health, in which there were no intergroup differences. Similar findings were found at the end of the second year.

\section{Discussion}

This study was undertaken in an attempt to compare the effect on the patient's disease, functional level, and adaptation to her disease of care by the general practitioner and his health care team, standard hospital outpatient attendance, and home visiting by a hospital occupational therapist. Although few intergroup differences have been found in the 2-year period following the minimum of 14 days' stay in a rheumatology unit, some interesting information has been obtained.

Disease activity as measured by the articular index and ESR was unchanged over the 2-year period, with patients maintaining the improvement achieved in hospital. This sustained control of disease activity was mirrored by the stabilised function assessed in a carefully standardised fashion. However, important improvements in some functions in all groups may have been missed. A more detailed assessment of the patients in one group showed significant differences between what they could do shortly before discharge and what they did do 10 days later in their own homes. Better results can be expected in ideal, standardised surroundings in which fatigue, psychosocial factors, and family demands are minimised and when the patient's expectation and confidence are increased by continuous attention, support, and encouragement (Wright and Owen, 1976). These findings emphasise the importance of assessing function in the patient's home and 
question the validity of attempting to assess function during brief visits to busy outpatient clinics (Sainsbury, 1973).

There were no changes in the numbers of patients with problems of isolation. This is disappointing, since the numbers registered as disabled with the social services departments increased in each of the 2 years, and the numbers of patients in contact with a wide range of domiciliary services and the frequency of these contacts increased in the first year. Further, it was particularly disturbing to find that the social services and other local authority support in a wide range of categories tended to be withdrawn during the second year. The results of functional testing show that this did not reflect improved function and independence. They imply that there is a considerable unsatisfied need for this type of support and that new requests for help are answered by withdrawal of help from those already receiving it in an indiscriminate fashion.

The results of the rates of supply and usage of aids and appliances were more satisfactory. There was little evidence of failure of supply. Although many of these aids were supplied by the hospital, an equal number were supplied promptly by the local authority domiciliary services, thus ensuring that the patient could immediately use aids for which they had been assessed and trained. These patterns of supply probably explain the satisfactorily high usage rate at the end of the first year, the rates being considerably higher than in a similar study in Leeds, when delays in provision by the local authority services after hospital assessment were often lengthy (Thornley et al., 1977; Chamberlain et al., 1978).

Although there were no intergroup differences in the various methods of assessment, the independent assessor considered that those patients visited by the hospital based therapist had received the best overall care. Although judged to be less satisfactory, attendance at routine outpatient clinics was significantly better than general practitioner care. Perhaps these trends may be reflected in changes in function and disease activity in the future. Certainly it is easy to imagine that a skilled therapist who is aware of the medical and surgical facilities and opportunities available in the hospital unit, who is in the best place to assess changes in function, and who is trained to provide a wide range of aids and other support could manage these patients very well. In general, patients much preferred to have continuing contact with the hospital service through either the therapist or the outpatient clinic, because they felt their problems and difficulties were understood by the hospital team. Undoubtedly regular medical contact is important, since this was the chief factor mentioned by those satisfied with general practitioner care. The need for continuing attention was highlighted by the finding that, despite improved knowledge of their disease and well controlled disease activity and function, approximately half the patients in each group continued to have a negative attitude to their disease, being disinclined to improve their condition or mobility.

It had been hoped that the results of this study would give guidance to those responsible for planning the continuing care of patients with a chronic, progressive disease. In retrospect, it might have been wiser to have attempted either a longer follow-up or to have done the study with outpatients, preferrably those attending the clinic for the first time. Most patients show improvement with inpatient treatment, and in some this will be sustained for 2 or more years (Duthie, 1967). Further, because patients were only randomly allocated to a treatment group on their last day in the ward, all had received the same treatment to that date, many appliances had already been issued, and community help and contacts had been arranged. Thus the capacity for intergroup differences to develop was limited, and it was only on new or increasing problems that the therapeutic and support efforts of the follow-up staff could be expected to make an impact.

It is clear that some patients can be managed satisfactorily by any of the 3 alternative methods. But if, as suggested by the independent assessor, continuing contact with the hospital team is important, then there are financial advantages in using therapists instead of an increasing number of doctors to handle the increasing outpatient load at rheumatological clinics and the ambulance service to get them there.

We thank the other members of our units, medical, paramedical, and secretarial, who contributed to the overall care of the patients and the administration of this study.

\section{References}

Brattstrom, M. (1975). Teamwork in the rehabilitation of patients with chronic rheumatoid arthritis. Annals of Clinical Research, 7, 230-236.

Camp, A. V. (1971). An articular index for the assessment of rheumatoid arthritis. Orthopaedics Oxford, 4, 39-45.

Chamberlain, M. A., Thornley, G., and Wright, V. (1978). Evaluation of aids and equipment for both and toilet. Rheumatology and Rehabilitation, 17, 187-194.

Duthie, J. J. R. (1967). Medical management and prognosis in rheumatoid arthritis. Scottish Medical Journal, 12, 96-106.

Feigenbaum, S. L., Kaplan, S. B., Masi, A. T., and Chandler, R. W. (1977). Early clinical features that predict outcome in rheumatoid arthritis: results of a prospective study using multivariable analytic techniques. Arthritis and Rheumatism, 20, 115. 
Firth, D., Roberts, M., Wright, J. G., Heath, P., and Wright, V. (1973). The value of health visitors in a rehabilitation unit. Rheumatology and Rehabilitation, 12, 143-147.

Haworth, R. J., and Hollings, E. M. (In press). Are hospital assessments of daily living activities valid? International Journal of Rehabilitation Medicine.

Hollings, E. M., and Haworth, R. J. (1978). Supply and use of aids and appliances. British Journal of Occupational Therapy, 41, 336-339.

Sainsbury, S. (1973). Occasional Papers of Social Administration, No. 54. C Bell and Sons: London.

Thornley, G., Chamberlain, M. A., and Wright, V. (1977).
Evaluation of aids and equipment for bath and toilet. British Journal of Occupational Therapy, 40, 243-246.

Vignos, P. J., Thompson, H. M., Katz, S., Moskowitz, R. W., Fink, S., and Svec, K. H. (1972). Comprehensive care and psychosocial factors in rehabiltation in chronic rheumatoid arthritis. Journal of Chronic Diseases, 25, 457-467.

Williams, R. G. A., Johnston, M., Willis, L. A., and Bennett, A. E. (1976). Disability: a model and measurement technique. British Journal of Preventive and Social Medicine, 30, 71-78.

Wright, V., and Owen, S. (1976). The effect of rheumatoid arthritis on the social situation of housewives. Rheumatology and Rehabilitation, 15, 156-160. 